\title{
ARTICLE
}

Clinical Study

\section{Bridging The Age Gap: observational cohort study of effects of chemotherapy and trastuzumab on recurrence, survival and quality of life in older women with early breast cancer}

\author{
Alistair Ring (D) ${ }^{1}$, Nicolò Matteo Luca Battisti ${ }^{1}{ }^{1}$, Malcolm W. R. Reed ${ }^{2}$, Esther Herbert ${ }^{3}$, Jenna L. Morgan ${ }^{4}$, Michael Bradburn $^{3}$, \\ Stephen J. Walters ${ }^{3}$, Karen A. Collins ${ }^{5}$, Sue E. Ward ${ }^{6}{ }^{6}$, Geoffrey R. Holmes ${ }^{6}$, Maria Burton ${ }^{5}$, Kate Lifford ${ }^{7}$, Adrian Edwards ${ }^{7}$, \\ Thompson G. Robinson ${ }^{8}$, Charlene Martin ${ }^{4}$, Tim Chater ${ }^{3}$, Kirsty J. Pemberton ${ }^{3}$, Alan Brennan ${ }^{6}$, Kwok Leung Cheung 9 , Annaliza Todd ${ }^{4}$, \\ Riccardo A. Audisio ${ }^{10}$, Juliet Wright ${ }^{2}$, Richard Simcock ${ }^{11}$, Tracey Green ${ }^{12}$, Deirdre Revell ${ }^{12}$, Jacqui Gath ${ }^{12}$, Kieran Horgan ${ }^{13}$, \\ Chris Holcombe ${ }^{14}$, Matthew C. Winter ${ }^{15}$, Jay Naik ${ }^{16}$, Rishi Parmeshwar ${ }^{17}$, Margot A. Gosney ${ }^{18}$, Matthew Q. Hatton ${ }^{15}$, \\ Alastair M. Thompson ${ }^{19}$, Lynda Wyld (iD) ${ }^{4}$ and on behalf of the Age Gap TMG
}

\begin{abstract}
BACKGROUND: Chemotherapy improves outcomes for high risk early breast cancer $(E B C)$ patients but is infrequently offered to older individuals. This study determined if there are fit older patients with high-risk disease who may benefit from chemotherapy. METHODS: A multicentre, prospective, observational study was performed to determine chemotherapy ( \pm trastuzumab) usage and survival and quality-of-life outcomes in EBC patients aged $\geq 70$ years. Propensity score-matching adjusted for variation in baseline age, fitness and tumour stage.

RESULTS: Three thousands four hundred sixteen women were recruited from 56 UK centres between 2013 and 2018 . Two thousands eight hundred eleven (82\%) had surgery. 1520/2811 (54\%) had high-risk EBC and 2059/2811 (73\%) were fit. Chemotherapy was given to $306 / 1100$ (27.8\%) fit patients with high-risk EBC. Unmatched comparison of chemotherapy versus no chemotherapy demonstrated reduced metastatic recurrence risk in high-risk patients(hazard ratio [HR] 0.36 [95\% Cl 0.19-0.68]) and in 541 age, stage and fitness-matched patients(adjusted HR 0.43 [95\% Cl 0.20-0.92]) but no benefit to overall survival (OS) or breast cancer-specific survival (BCSS) in either group. Chemotherapy improved survival in women with oestrogen receptor (ER)-negative cancer (OS: HR 0.20 [95\% Cl 0.08-0.49];BCSS: HR 0.12 [95\% Cl 0.03-0.44]).Transient negative quality-of-life impacts were observed. CONCLUSIONS: Chemotherapy was associated with reduced risk of metastatic recurrence, but survival benefits were only seen in patients with ER-negative cancer. Quality-of-life impacts were significant but transient.

TRIAL REGISTRATION: ISRCTN 46099296
\end{abstract}

British Journal of Cancer (2021) 125:209-219; https://doi.org/10.1038/s41416-021-01388-9

\section{BACKGROUND}

In 2014-2016 over 18,500 women per year aged $\geq 70$ years were diagnosed with breast cancer in the UK, representing $34 \%$ of all diagnoses. ${ }^{1}$ Breast cancer survival is worse in older patients ${ }^{2}$ who have not experienced similar outcome improvements compared with younger individuals in the past three decades. ${ }^{3}$ This may reflect late presentation, more comorbidities or undertreatment.
Significant treatment variations between centres are frequently reported in older adults. ${ }^{4,5}$ However, interpreting such data can be challenging without information on fitness, which may mitigate treatment benefits, due to competing mortality risks and increased treatment-related toxicity.

Chemotherapy benefit in older women is controversial. While there have been many high-quality randomised clinical trials

\footnotetext{
1Department of Medicine, Breast Unit, The Royal Marsden Hospital NHS Foundation Trust, London, UK \& Breast Cancer Research Division, The Institute of Cancer Research, London, UK; ${ }^{2}$ Brighton and Sussex Medical School, Brighton, UK; ${ }^{3}$ Clinical Trials Research Unit, School for Health and Related Research, University of Sheffield, Sheffield, UK; ${ }^{4}$ Department of Oncology and Metabolism, University of Sheffield Medical School, Sheffield, UK; ${ }^{5}$ College of Health, Wellbeing and Life Sciences, Department of Allied Health

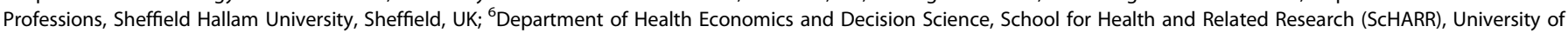
Sheffield, Sheffield, UK; ${ }^{7}$ Division of Population Medicine, Cardiff University, Cardiff, UK; ${ }^{8}$ Department of Cardiovascular Sciences and NIHR Biomedical Research Centre, University

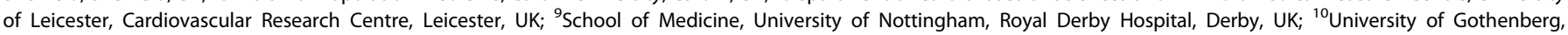
Sahlgrenska Universitetssjukhuset, Göteborg, Sweden; ${ }^{11}$ Sussex Cancer Centre, Royal Sussex County Hospital, Brighton, UK; ${ }^{12}$ Yorkshire and Humber Consumer Research Panel,

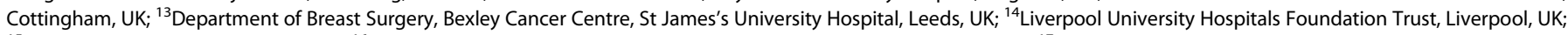

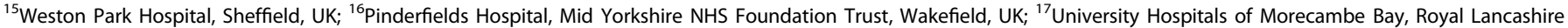
Infirmary, Lancaster, Lancashire, UK; ${ }^{18}$ Royal Berkshire NHS Foundation Trust, Reading, UK and ${ }^{19}$ Department of Surgery, Baylor College of Medicine, Houston, TX, USA Correspondence: Lynda Wyld (I.wyld@sheffield.ac.uk)
}

These authors contributed equally: Alistair Ring, Nicolò Matteo Luca Battisti.

Members of the Age Gap TMG are listed above Acknowledgements.

Received: 29 October 2020 Revised: 20 February 2021 Accepted: 31 March 2021

Published online: 10 May 2021 
(RCTs) to evaluate the impact of systemic chemotherapy, the majority of trials excluded or recruited poorly amongst older patients, and tended to enrol fitter individuals. ${ }^{6}$ This reflects clinicians' and patients' toxicity concerns and reticence from trialists about diluting the study power by introducing higher morbidity rates and competing causes of death in less fit older patients.

Older adults derive less benefit from chemotherapy compared to younger patients. Benefit is present between the ages of 70 and 80 , although data for women aged over 80 years are scarce. ${ }^{7}$ The Bridging the Age Gap study was designed to recruit a large, realworld, cohort of older women with breast cancer including detailed baseline fitness data and information about the cancer, treatment received and outcomes. The objectives of this study analysis were to determine health status-stratified outcomes for EBC patients aged $\geq 70$ according to whether they received guideline concordant or non-concordant care with a particular focus on chemotherapy use. In this paper, the age- and risk-stratified patterns of receipt of adjuvant systemic therapy are described in older EBC patients, with propensity score-matched analysis of disease recurrence, survival and quality-of-life outcomes.

\section{METHODS}

\section{Study design}

Bridging the Age Gap is a prospective multicentre, observational cohort study. Patients were recruited from 56 UK centres in England and Wales (Supplementary Table 1). Eligible patients were women $\geq 70$ years at diagnosis of primary operable invasive breast cancer (TNM stages: T1-3 (plus some T4b), N0-1, M0). Those unsuitable for surgery or with previous $\mathrm{EBC}$ within five years were not eligible.

\section{Baseline data collection}

Patients were recruited at the time of EBC diagnosis and before commencing treatment and could participate at three levels: full, partial (no requirement to complete quality of life [QoL] assessments) or by proxy (simple third-party data collection for those with cognitive impairment).

Baseline data were collected about the primary tumour including; cancer type, grade, nodal status, tumour size, oestrogen $(E R)$, progesterone (PR) and human epidermal growth factor receptor 2 (HER2) status. Staging was performed if clinically indicated. Surgical, radiotherapy and systemic therapy data were collected.

At baseline, patients underwent assessments using validated tools including: comorbidities (Charlson comorbidity index [CCI]), ${ }^{8}$ nutrition (Abridged Patient Generated Subjective Global Assessment [aPG-SGA]), 9,10 functional status (Activities of Daily Living $[A D L]),{ }^{11}$ advanced functional status (Instrumental Activities of Daily Living [IADL]), ${ }^{12}$ dementia (Mini Mental State Examination [MMSE]), ${ }^{13}$ Eastern Cooperative Oncology Group Performance Status (ECOG PS) and medication list.

Quality-of-life was assessed using the EuroQol-5D-5L (EQ-5D$5 \mathrm{~L}) .{ }^{14}$ Assessments on the European Organisation for the Research and Treatment of Cancer QoL Questionnaire (EORTC-QLQ)-C30, ${ }^{15}$ EORTC-QLQ-BR23, ${ }^{16}$ EORTC-QLQ-ELD15 ${ }^{17}$ were also collected but are presented elsewhere. ${ }^{18}$

\section{Follow-up and outcomes}

Patients were followed up at 6 weeks, and 6, 12, 18 and 24 months. Survival outcomes (date and cause of death) were obtained at 52 months median follow-up from the UK cancer registry. All patients were assessed for recurrence and QoL at each visit. Complications were categorised using the Common Terminology Criteria for Adverse Events system (CTCAE v4.0).

Chemotherapy-related mortality was defined as death within 30 days of chemotherapy or if chemotherapy was documented as a contributing cause. Deaths were categorised as disease related or other causes. Deaths were reviewed by the chief investigator blind to treatment decisions. Deaths were classified as disease related if the death was related to the initial breast cancer. Patients for whom the cause could not be established were excluded from cause-specific analyses.

Statistical analyses

Analyses were performed in IBM SPSS statistics version 24 and $R$ version 3.6.3. ${ }^{19} \mathrm{~A} p<0.05$ was considered statistically significant.

The relationships between systemic therapy use and tumour and patient characteristics were evaluated using uni- and multivariable logistic regression. High-risk EBC was defined if any of the following criteria were present: node-positive, ER-negative, HER2 positive, grade 3 or Recurrence Score $\geq 25$. (Supplementary Table 2a). Additional analyses were conducted in patients with ER-negative and HER2-positive tumours, where the benefits from chemotherapy might be anticipated. Fitness was defined based on geriatric assessments and categorised into fit, vulnerable and frail according to a cumulative score including measures of functional status, comorbidities, polypharmacy, nutritional status and cognitive status (Supplementary Table 2b).

Both overall survival (OS) and breast cancer-specific survival (BCSS) were compared in treated and untreated patients. A Cox proportional hazards model was fitted using regression-based adjustment based on covariates of: treatment; age; categories of aPG-SGA, ADL, IADL, CCI, MMSE, ECOG, medications and Nottingham Prognostic Index (NPI) ${ }^{20}$ and HER2 for all high-risk patients. Hazard ratios (HR) and corresponding 95\% confidence intervals (Cls) were calculated.

A propensity score adjustment among sufficiently similar highrisk patients was fitted using a Cox model with a shared frailty term (or random effect) for matched patients. Participants were matched exactly on NPI category and HER2 status, and logistic regression was used to calculate propensity scores for treatment in relation to age, aPG-SGA category, ADL category, IADL category, MMSE category, CCI category, ECOG PS category and number of medications. The ratio and calliper widths of the propensity scores were chosen following examination of the propensity score overlaps for several combinations of ratios and callipers. A 1:3 ratio for chemotherapy versus no chemotherapy and a calliper of 0.25 times the propensity scores' standard deviation was used to ensure participants were closely matched whilst retaining as many patients as possible.

The QoL questionnaires were scored according to the EQ-5D$5 \mathrm{~L}$ User Guide (Version 3.0). ${ }^{21}$ Missing data were managed accordingly. The QoL analysis included only patients with highrisk EBC as detailed in Supplementary Table $2 a$ and where questionnaires were available. The mean difference $(95 \% \mathrm{Cl})$ of the domain scores at each time-point, adjusted for baseline scores, was calculated with linear regression models for high-risk participants. Propensity score-matching was also performed, as detailed above, to compare the EQ-5D-5L usual activities score in a matched cohort receiving chemotherapy versus patients not receiving it.

\section{RESULTS}

Between January 2013 and June 2018, 3456 women were recruited from 56 centres in England and Wales. This analysis was restricted to the 2811 women who underwent surgery within 6 months of diagnosis (STROBE diagram [Fig. 1]). ${ }^{22}$ Patients' characteristics according to geriatric assessments, tumour characteristics, postoperative histology and surgery performed are shown in Table 1.

Of the 2811 patients, $397(14.1 \%)$ received chemotherapy (365 [92\%] in the adjuvant setting, 30 [8\%] in neoadjuvant setting, and 2 [0.5\%] unknown). Of those 380 patients for whom the 


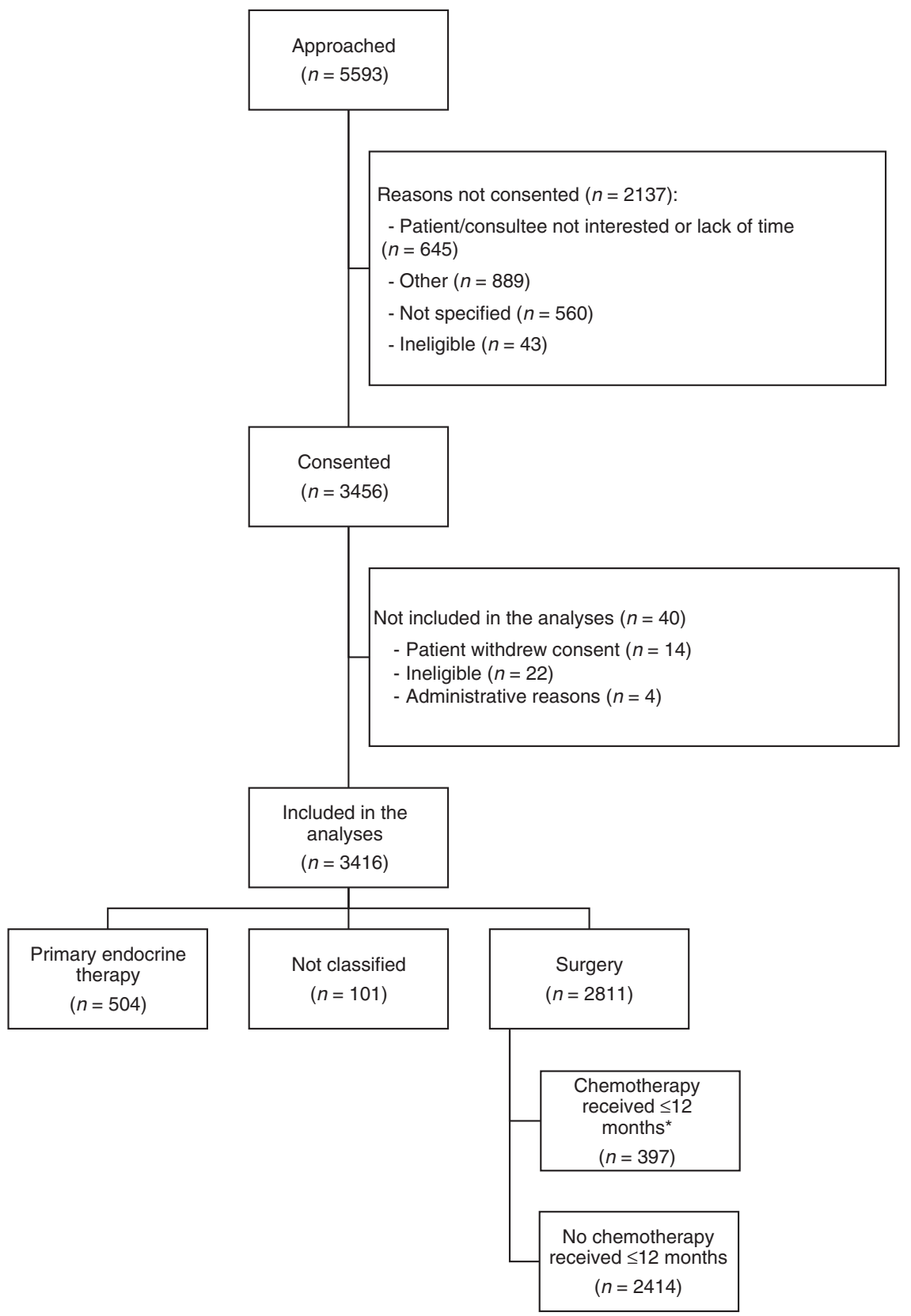

* Patients who only received palliative chemotherapy regimens where not counted as having received chemotherapy.

Fig. 1 STROBE diagram. STROBE flow diagram for the chemotherapy vs no chemotherapy analyses.

chemotherapy regimen received was known, $132(34.7 \%)$ received an anthracycline-taxane combination, 124 (32.6\%) a taxane (without anthracycline), 123 (32.4\%) an anthracycline and 1 CMF. 332 patients (11.8\%) had HER2-positive EBC. Of these patients, 150 (45.1\%) received chemotherapy plus trastuzumab, 13 (3.9\%) trastuzumab without chemotherapy, and $9(2.7 \%)$ chemotherapy without trastuzumab. Overall, 1753/2811 (62.4\%) patients received radiotherapy and 2239/2354 (95.1\%) ER-positive patients received endocrine therapy.

Chemotherapy receipt according to tumour and patient characteristics is shown in Supplementary Tables 3 and 4 . Univariate and multivariate analyses are shown in Table 2. Younger, less dependent patients with high-risk tumours and with fewer comorbidities were more likely to receive chemotherapy.
High-risk tumours were present in 1520 (54\%) patients and 376/ 1520 (25\%) received chemotherapy compared with 21/1291 (1.6\%) of patients with non-high-risk tumours (Table 3a). 2059 patients (73\%) were fit and 752 vulnerable or frail (27\%) (Table $3 b)$. Of those who were fit, 1100 also had high-risk EBC, and of these patients $306(28 \%)$ received chemotherapy (Table 3c).

At a median follow-up of 52 months, mortality status was available for $98 \%(1495 / 1520)$ of high-risk patients (371 in the chemotherapy group, 1124 in the no chemotherapy group). Chemotherapy was associated with a longer OS, but the difference was not statistically significant when adjusted for other covariates (unadjusted HR 0.55 [95\% Cl 0.40-0.73, $p<0.001]$ and adjusted HR 0.87 [95\% Cl $0.58-1.28, p=0.469$ ] (Fig. 2a). In a propensity scorematched analysis 200 patients receiving chemotherapy were 
Table 1. Baseline tumour, patient and treatment characteristics by age.

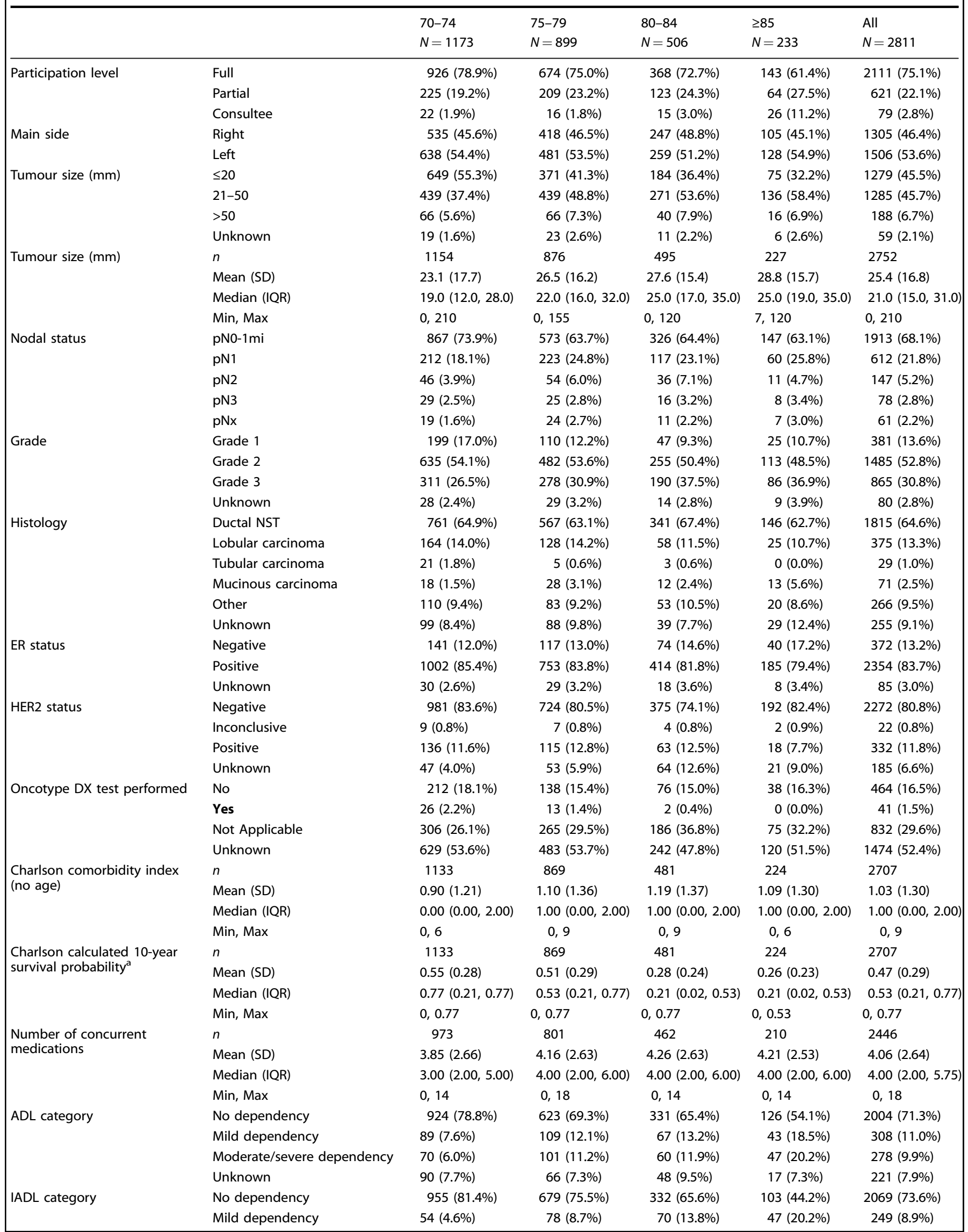




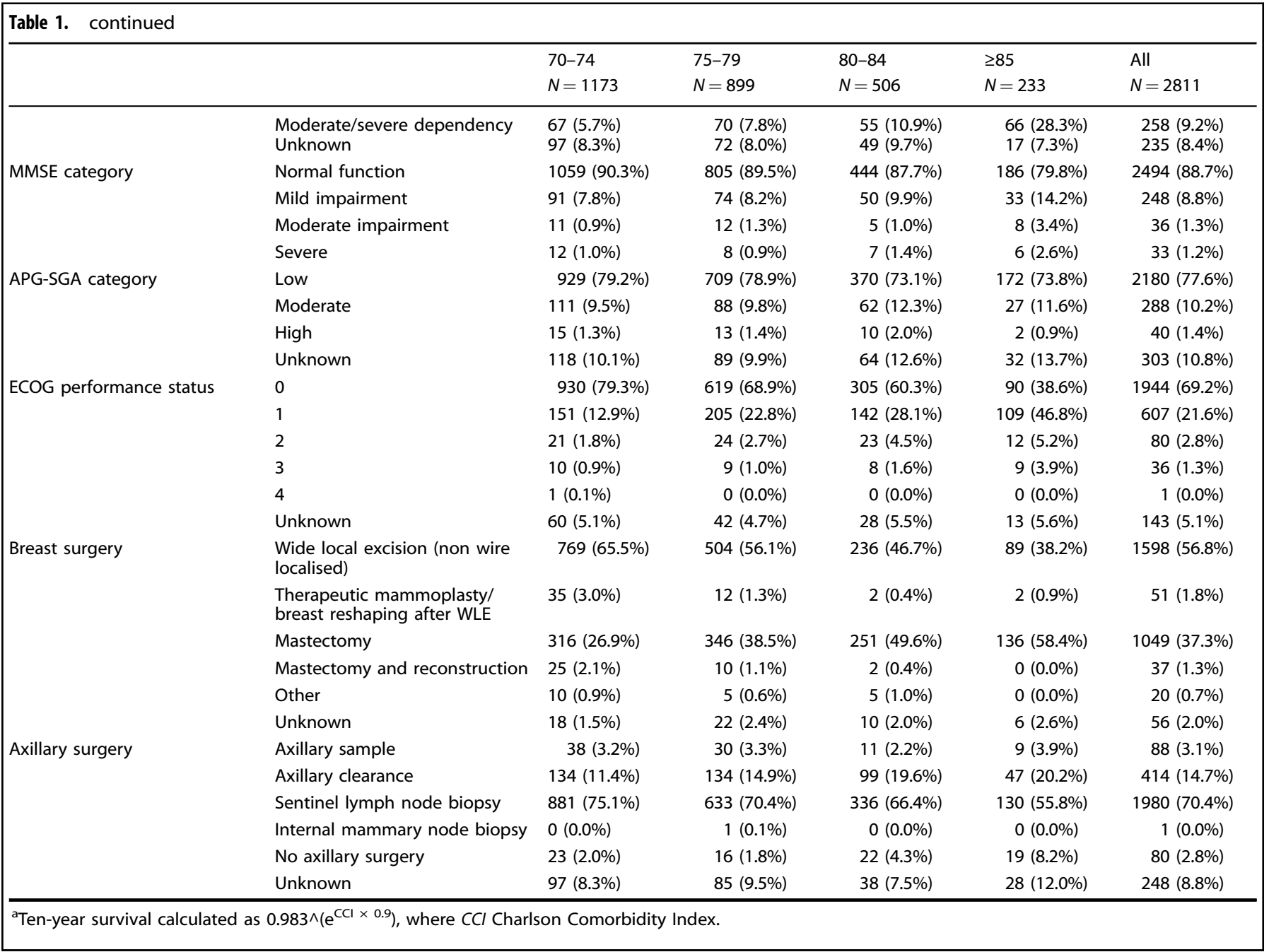

matched to 350 who did not receive it. Supplementary Table 5 shows the characteristics of the matched dataset and the matching process and quality are summarised in Supplementary Fig. 1. Mortality status was available for 542 (99\%) of the matched patients. Chemotherapy was associated with a longer OS although this was not statistically significant (HR 0.79 [95\% Cl 0.50-1.26, $p=$ 0.320]) (Fig. 2b).

BCSS was available for $98 \%(1486 / 1520)$ of patients in the highrisk population. Chemotherapy was not associated with improved BCSS (unadjusted HR 0.76 [95\% Cl 0.53-1.10, $p=0.147]$ and adjusted HR 0.92 [95\% Cl 0.56-1.53, $p=0.758]$ ) (Fig. 2c). In the propensity score-matched population, BCSS was available for 539 patients (98\%). Chemotherapy was also not found to be associated with improved BCSS (HR 0.93 [95\% Cl 0.52-1.66, $p=$ 0.798]) (Fig. 2d).

Metastatic recurrence data were available for 1498 high-risk patients (99\%). Chemotherapy was associated with a significantly lower risk of metastatic recurrence in the unmatched population (unadjusted HR 0.67 [95\% Cl 0.43-1.04, $p=0.077$ ] and adjusted HR 0.36 [95\% Cl 0.19-0.68, $p=0.002]$ ) (Fig. 2e). In 541 matched patients $(98 \%)$, chemotherapy was also associated with a lower metastatic recurrence risk (HR $0.53[95 \% \mathrm{Cl} 0.26-1.07, p=0.076]$ ) (Fig. 2f).

Additional post-hoc exploratory analyses were performed in disease subgroups. Out of 369 patients with ER-negative EBC and known mortality status, 132 (35.8\%) received chemotherapy. In a propensity score-matched analysis in 136 patients, chemotherapy was associated with better OS (HR 0.20 [0.08-0.49]) and BCSS (HR 0.12 [0.03-0.44]) (Fig. 3, Supplementary Table 6 and Supplementary Fig. 2). Three hundred twenty six patients with HER2-positive EBC and known mortality status of whom 156 (47.9\%) received chemotherapy with or without trastuzumab. Fewer deaths from breast cancer and other causes occurred in those receiving chemotherapy with or without trastuzumab. However, in a matched analysis in 137 patients, the differences were not statistically significant for OS (HR 0.63 [0.27-1.48]) or BCSS (HR 0.50 ([0.16-1.63]) (Fig. 3, Supplementary Table 6 and Supplementary Fig. 2).

Supplementary Table 7 outlines chemotherapy toxicity. Among 397 patients receiving chemotherapy, there was one chemotherapy-related death $(0.25 \%)$ (due to congestive heart failure) and 132 (33.2\%) had an episode of infection, which was grade 3 or 4 in 50 (12.6\%). Among the 163 patients who received trastuzumab, $4(2.5 \%)$ experienced cardiac failure within the first 6 months and $12(6.7 \%)$ within the first year.

Among 2811 patients undergoing surgery, the QoL analysis was restricted to $1520 / 2811$ (54.1\%) with high-risk EBC of whom 1315/ $1520(86.5 \%)$ had an EQ-5D-5L score available at baseline. Of these patients, 376/1520 (24.7\%) received chemotherapy. Health utilities were similar with estimated mean differences less than 0.02 units $(p>0.1)$, whereas the visual analogue scale (VAS) measures were significantly worse at 6 months in patients receiving chemotherapy versus not (adjusted mean difference $-6.57,95 \% \mathrm{Cl}-8.74$ to -4.40 , $p<0.001)$. Changes were no longer significant at 12 months and thereafter (Supplementary Table 8; Supplementary Fig. 3). 
Table 2. Relationship between chemotherapy use and patient characteristics: univariate (Table 2a) and multi-variable (Table $2 \mathrm{~b}$ ) analyses.

\begin{tabular}{|c|c|c|c|}
\hline Variable & Level & OR $(95 \% \mathrm{Cl})$ & $P$-value \\
\hline Age & & $0.84(0.82,0.87)$ & $<0.001$ \\
\hline ADL score & & $1.07(1.04,1.11)$ & $<0.001$ \\
\hline IADL score & & $1.77(1.43,2.25)$ & $<0.001$ \\
\hline $\mathrm{CCl}$ (no age) & & $0.84(0.77,0.93)$ & $<0.001$ \\
\hline APG-SGA & & $0.95(0.89,1.01)$ & 0.127 \\
\hline Allred score & & $0.80(0.78,0.83)$ & $<0.001$ \\
\hline \multirow[t]{3}{*}{ Tumour grade } & Grade 1 & - & - \\
\hline & Grade 2 & $9.04(3.78,29.58)$ & $<0.001$ \\
\hline & Grade 3 & $37.67(15.87,122.76)$ & $<0.001$ \\
\hline ER positive & & $0.22(0.17,0.28)$ & $<0.001$ \\
\hline \multirow[t]{2}{*}{ HER2 status ${ }^{a}$} & Negative & - & - \\
\hline & Positive & $8.49(6.57,10.97)$ & $<0.001$ \\
\hline \multirow[t]{4}{*}{ MMSE category } & Normal function & - & - \\
\hline & Mild impairment & $0.75(0.49,1.11)$ & 0.172 \\
\hline & Moderate impairment & $1.18(0.44,2.67)$ & 0.711 \\
\hline & Severe & $0.38(0.06,1.27)$ & 0.188 \\
\hline \multirow[t]{4}{*}{ Nodal status ${ }^{\mathrm{b}}$} & pNo-1mi & - & - \\
\hline & $\mathrm{pN} 1$ & $2.18(1.69,2.80)$ & $<0.001$ \\
\hline & $\mathrm{pN} 2$ & $5.05(3.47,7.29)$ & $<0.001$ \\
\hline & pN3 & $6.42(3.96,10.30)$ & $<0.001$ \\
\hline
\end{tabular}

(b) Results from the multi-variable logistic regression model.

\begin{tabular}{|c|c|c|c|}
\hline Variable & Level & OR $(95 \% \mathrm{Cl})$ & $P$-value \\
\hline IADL score & & $1.97(1.53,2.63)$ & $<0.001$ \\
\hline \multirow[t]{3}{*}{ Tumour grade } & Grade 1 & - & - \\
\hline & Grade 2 & $8.42(3.05,34.90)$ & $<0.001$ \\
\hline & Grade 3 & $29.50(10.59,123.00)$ & $<0.001$ \\
\hline \multirow[t]{2}{*}{ HER2 status } & Negative & - & - \\
\hline & Positive & $8.94(6.19,13.01)$ & $<0.001$ \\
\hline \multirow[t]{3}{*}{ Nodal status } & pNo-1mi & & \\
\hline & $\mathrm{pN} 1$ & $4.01(2.81,5.75)$ & $<0.001$ \\
\hline & $\mathrm{pN} 2$ & $11.24(6.43,19.74)$ & $<0.001$ \\
\hline
\end{tabular}

A similar pattern on EQ-5D-5L usual activities score was seen in 520 propensity score-matched patients (including 118 patients receiving chemotherapy and 332 not receiving it) (Supplementary Fig. 4).

\section{DISCUSSION}

This study represents one of the largest prospective cohort studies conducted in older women with breast cancer and provides valuable data on tumour characteristics and health of older EBC patients. As expected, the majority of patients had relatively good prognosis tumours, with relatively low rates of nodal involvement and adverse biology as determined by ER and HER2 status.
Nonetheless, there remained a substantial proportion of high risk, fit patients (on baseline assessments), with a high relapse risk in their expected lifetime. Ensuring that these patients receive adequate treatment is a priority for clinicians.

A key finding of this study is that $27.8 \%$ of fit high-risk EBC older patients received chemotherapy. In the ACheW study $30 \%$ of highrisk EBC patients were offered chemotherapy and $17 \%$ received it. ${ }^{23}$ Analyses of European and US registry data report similar findings. ${ }^{5,24,25}$ These analyses did not consider recurrence risk (as determined by histopathological variables) and patients' fitness (to not only receive treatment but also to live long enough to benefit). The current study overcame these limitations, by defining recurrence risk and fitness, and still demonstrates low 
Table 3. Chemotherapy use according to risk of recurrence and fitness.

(a) Use of chemotherapy by risk status.

\begin{tabular}{|c|c|c|c|}
\hline Risk & Chemotherapy & No Chemotherapy & Total \\
\hline High risk & $376(24.7 \%)$ & $1144(75.3 \%)$ & $1520(100.0 \%)$ \\
\hline Non-high risk & 21 (1.6\%) & $1270(98.4 \%)$ & $1291(100.0 \%)$ \\
\hline Total & 397 (14.1\%) & 2414 (85.9\%) & 2811 (100.0\%) \\
\hline
\end{tabular}

(b) Use of chemotherapy by fitness.

\begin{tabular}{lcrr}
\hline Fitness & Chemotherapy & No Chemotherapy & \multicolumn{1}{c}{ Total } \\
\hline Fit & $322(15.6 \%)$ & $1737(84.4 \%)$ & $\mathbf{2 0 5 9}(\mathbf{1 0 0 . 0 \% )}$ \\
Vulnerable & $75(10.0 \%)$ & $675(90.0 \%)$ & $\mathbf{7 5 0}(\mathbf{1 0 0 . 0 \% )}$ \\
Frail & $0(0.0 \%)$ & $2(100.0 \%)$ & $\mathbf{2 ( 1 0 0 . 0 \% )}$ \\
Total & $\mathbf{3 9 7}(\mathbf{1 4 . 1 \% )}$ & $\mathbf{2 4 1 4}(\mathbf{8 5 . 9 \% )}$ & $\mathbf{2 8 1 1}(\mathbf{1 0 0 . 0 \% )}$
\end{tabular}

(c) Use of chemotherapy by risk and fitness.

\begin{tabular}{|c|c|c|c|c|c|}
\hline \multirow[t]{2}{*}{ Fitness } & \multicolumn{2}{|l|}{ High risk } & \multicolumn{2}{|l|}{ Non-high risk } & \multirow[t]{2}{*}{ Total } \\
\hline & Chemotherapy & No chemotherapy & Chemotherapy & No chemotherapy & \\
\hline Fit & $306(14.9 \%)$ & 794 (38.6\%) & $16(0.8 \%)$ & $943(45.8 \%)$ & 2059 (100.0\%) \\
\hline Frail & $0(0.0 \%)$ & $1(50 \%)$ & $0(0.0 \%)$ & $1(50 \%)$ & $2(100 \%)$ \\
\hline Total & 376 (13.4\%) & 1144 (40.7\%) & $21(0.7 \%)$ & 1270 (45.2\%) & 2811 (100.0\%) \\
\hline
\end{tabular}

Bold values represent the total numbers for each column or row.

chemotherapy uptake. This may be due to uncertainty on chemotherapy benefit in older adults, toxicity concerns and patients' and carers' choice.

In order to investigate the survival benefits of chemotherapy for older EBC patients, we conducted survival analyses in those at high risk of recurrence. Ideally this question should be addressed by RCTs. Recruiting older patients into RCTs comparing different chemotherapy regimens is feasible, ${ }^{26}$ but trials comparing chemotherapy with no chemotherapy have failed to recruit. ${ }^{27,28}$ Moreover, older patients enrolled in RCTs may be fitter and not necessarily representative of a real-world population. ${ }^{6}$ In contrast, this cohort study recruited well, and recruited patients with a broad fitness range.

Our analyses attempted to correct for confounders, specifically the fact that younger, fitter patients might be more likely to receive chemotherapy, but also are biologically more likely to survive longer irrespective of chemotherapy effect. This effect is perhaps most apparent when comparing the unmatched and matched OS analyses (Fig. 2a, b).

In the high-risk population chemotherapy reduced the risks of metastatic recurrence, which did not translate into better survival. This may be because the benefit was modest and the fact that median OS for ER-positive metastatic disease patients often exceeds 3 years with contemporary therapies. ${ }^{29}$ Irrespective, a reduction in metastatic relapses, with their symptomatic, psychological and financial implications, may be sufficient grounds on which to offer treatment even in the absence of a survival benefit. Longer term follow-up will be required to further explore this.

Chemotherapy benefits are small for most ER-positive, HER2negative EBC patients. Therefore, we performed exploratory analyses in patients with the more chemotherapy-sensitive subtypes, i.e. ERnegative and HER2-positive disease. In ER-negative EBC patients there was an apparent reduction of breast cancer deaths with chemotherapy. These data are consistent with an US SEER analysis suggesting that adjuvant chemotherapy benefit in older patients were restricted to those with ER-negative disease. ${ }^{28,30}$ In HER2positive EBC patients, fewer breast cancer deaths occurred in those who received chemotherapy with or without trastuzumab although the differences were not statistically significant in a matched analysis. This could be explained by the small numbers in this subgroup analysis. However, a retrospective study demonstrated that HER2-positive EBC older patients do not have inferior long-term outcomes compared with younger adults not receiving chemotherapy. ${ }^{31}$ Low Ki67 and high bcl2 expression in the older cohort of HER2-positive patients might explain this better prognosis and also relative chemo-resistance. ${ }^{31}$

Our study found that mortality rates from chemotherapy were very low and side effects consistent with previous analyses. ${ }^{32}$ Follow-up of the cohort is planned at 10 years and may provide data about longer term benefits, although it should be recognised that with longer follow-up competing mortality causes are likely have a greater impact.

Our analysis also demonstrates that chemotherapy has a significant negative impact at 6 months on QoL, which is a meaningful endpoint in the context of a more limited survival benefit and increased risk of toxicities in this population. However, this effect resolves at 12 months consistent with previous findings in smaller or younger cohorts of patients ${ }^{33,34}$ and is described in a more extensive analysis performed on this patient cohort. $^{18}$

A key strength of this study is that patients were recruited from a broad range of academic and general centres across the UK, and were likely to reflect contemporary practice and outcomes. However, despite the inclusive entry criteria and low level of intervention there was still the possibility of selection bias. In a separate analysis of this study we found that patients who did not enter the trial following screening were older and had worse functional ability. ${ }^{35}$ Also, as patients were not randomised, unmeasured variables might have influenced our findings despite 

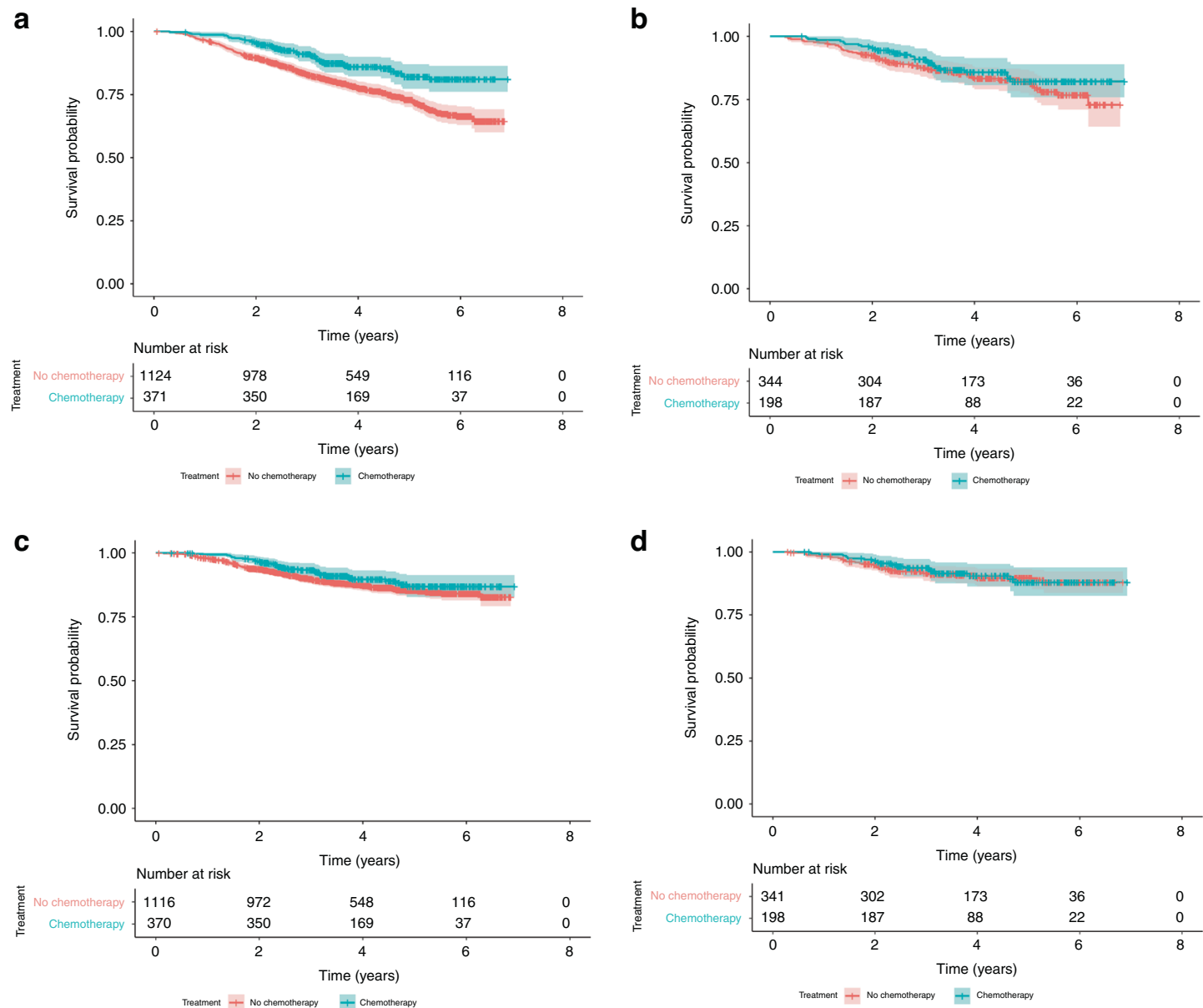

e

$\mathbf{f}$
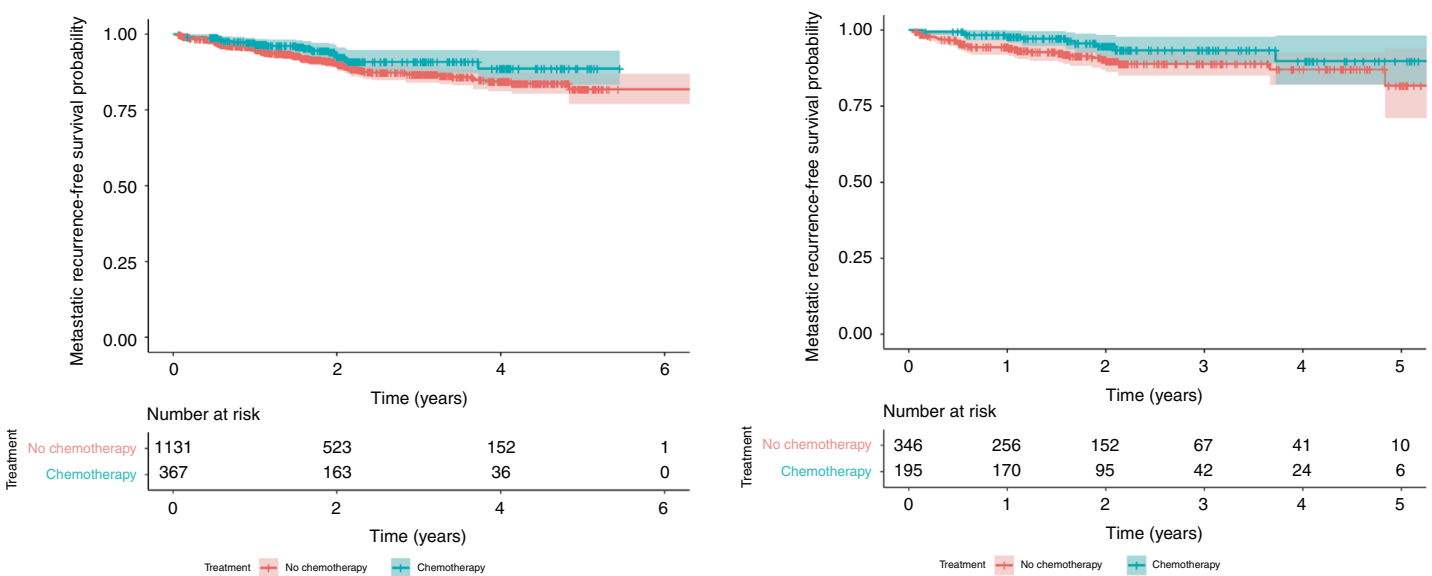

Fig. 2 Kaplan-Meier plots of survival and metastatic recurrence outcomes. a Overall Survival in unmatched high-risk patients $(n=1495)$. Adjusted HR 0.87 (95\% Cl 0.58-1.28, $p=0.47$ ). b Overall survival in matched high-risk patients $(n=542)$. Adjusted HR 0.79 (95\% Cl: 0.50-1.26, $p=0.32)$. c Breast cancer-specific survival in unmatched high-risk patients $(n=1486)$. Adjusted HR $0.92(95 \% \mathrm{Cl}: 0.56-1.53, p=0.76)$. d Breast cancer-specific survival in matched high-risk patients $(n=539)$. Adjusted HR 0.93 (95\% Cl: $0.52-1.66, p=0.80)$. e Metastatic recurrence in unmatched high-risk patients $(n=1498)$. Adjusted HR 0.36 ( $95 \% \mathrm{Cl}: 0.19-0.68, p=0.002)$. f Metastatic recurrence in matched high-risk patients $(n=541)$. Adjusted HR 0.53 (95\% Cl: 0.26-1.07, $p=0.08$ ).

propensity score matching. The extent to which these data reflect practice and outcomes outside of the UK is unknown, although some published data do appear comparable. ${ }^{24,25}$

In summary, this study demonstrates that there are a significant number of older but fit patients with high-risk EBC who are not receiving adjuvant chemotherapy. Some of these patients, particularly those with ER-negative disease, may derive benefit from chemotherapy. Clearly the benefits need to be discussed in the context of potential side effects and the transient negative impact on QoL. Nonetheless, it is important that individualised treatment decisions and discussions are made to ensure the best outcomes for older adults. 

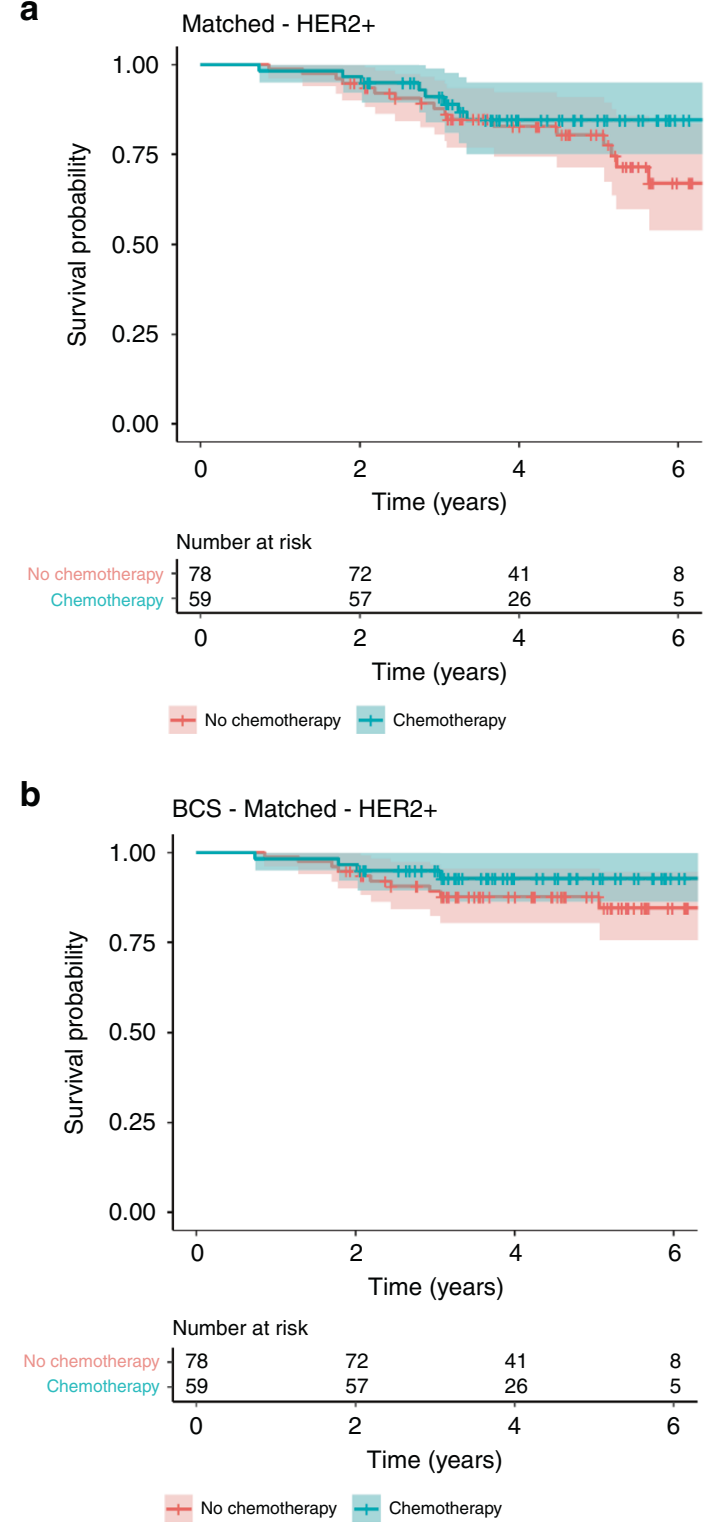

Matched - ER-

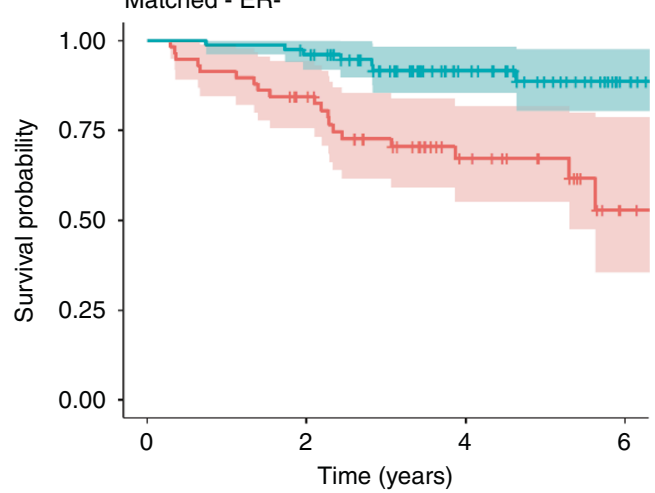

Number at risk

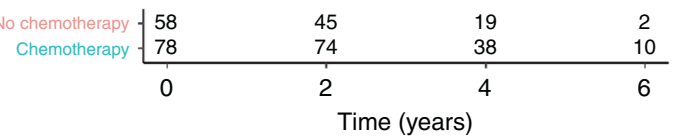

+ No chemotherapy + Chemotherapy

BCS - Matched - ER-

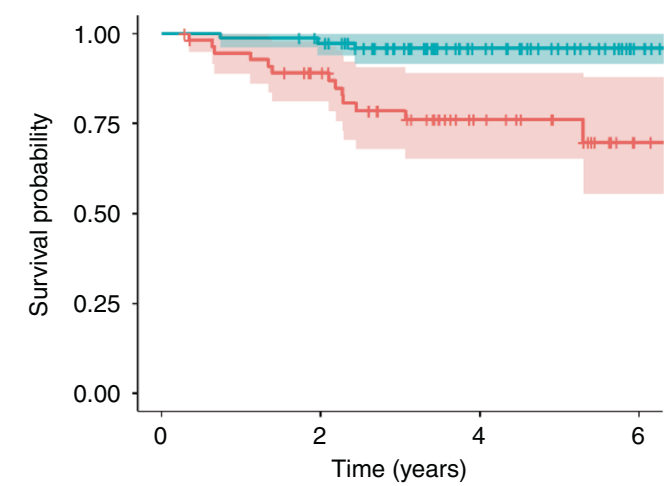

Number at risk

$\begin{gathered}\text { No chemotherapy } \\
\text { Chemotherapy }\end{gathered}=$\begin{tabular}{cccc}
57 & 44 & 19 & 2 \\
78 & 74 & 38 & 10 \\
\hline 0 & 2 & 4 & 6
\end{tabular}

+ No chemotherapy + Chemotherapy

Fig. 3 Kaplan-Meier plots for survival outcomes in matched patients with HER2-positive or ER-negative breast cancer. a Overall survival in patients with HER2-positive breast cancer $(n=137)$ : HR 0.63 [0.27-1.48]; and in patients with ER-negative breast cancer $(n=136)$ : HR 0.20 [0.08-0.49]. b Breast cancer-specific survival in patients with HER2-positive breast cancer $(n=137)$ : HR 0.5 [0.16-1.63]; and in patients with ERnegative breast cancer $(n=135)$ : HR 0.12 [0.03-0.44].

\section{AGE GAP TMG}

Lynda Wyld ${ }^{4}$, Malcolm WR Reed ${ }^{2}$, Karen Collins ${ }^{20}$, Sue Ward ${ }^{6}$, Geoff Holmes ${ }^{6}$, Jenna Morgan $^{4}$, Mike Bradburn ${ }^{3}$, Stephen Walters ${ }^{3}$, Maria Burton ${ }^{20}$, Kate Lifford ${ }^{21}$, Adrian Edwards $^{21}$, Kate Brain ${ }^{21}$, Alistair Ring ${ }^{22,23}$, Esther Herbert ${ }^{3}$, Thomson Robinson ${ }^{24}$, Charlene Martin ${ }^{4}$, Tim Chater ${ }^{3}$, Kirsty Pemberton ${ }^{3}$, Anne Shrestha ${ }^{4}$, Anthony Nettleship ${ }^{25}$, Paul Richards ${ }^{6}$, Alan Brennan ${ }^{6}$, Kwok Leung Cheung ${ }^{26}$, Annaliza Todd ${ }^{4}$, Helena Harder ${ }^{2}$, Riccardo Audisio ${ }^{10}$, Nicolò Matteo Luca Battisti ${ }^{22,23}$, Juliette Wright ${ }^{2}$, Richard Simcock ${ }^{2}$, Chris Murray ${ }^{25}$, Alistair M Thomson ${ }^{19}$, Margot Gosney ${ }^{27}$, Matthew Hatton $^{15}$, Fiona Armitage ${ }^{15}$, Julietta Patnick ${ }^{28}$, Tracy Green ${ }^{29}$, Deirdre Revill ${ }^{29}$, Jacqui Gath $^{29}$, Kieran Horgan ${ }^{30}$, Chris Holcombe ${ }^{31}$, Matt Winter ${ }^{15}$, Jay Naik ${ }^{16}$, Rishi Parmeshwar ${ }^{17}$

${ }^{20}$ Faculty of Health and Wellbeing, Department of Allied Health Professions, Sheffield Hallam University, Collegiate Cresent Campus, Sheffield, UK; ${ }^{21}$ Division of Population Medicine, Cardiff University, Heath Park, Cardiff, UK; ${ }^{22}$ Breast Unit - Department of Medicine - The Royal Marsden NHS Foundation Trust, London, UK; ${ }^{23}$ Breast Cancer Research Division, The Institute of Cancer Research, London, UK; ${ }^{24}$ Department of Cardiovascular Sciences, University of Leicester, Cardiovascular Research Centre, Glenfield General Hospital, Leicester, UK; ${ }^{25}$ EpiGenesys, University of Sheffield, Sheffield, UK; ${ }^{26}$ University of Nottingham, Royal Derby Hospital, Derby, UK; ${ }^{27}$ School of Psychology \& Clinical Language Sciences, University of Reading, Reading, UK; ${ }^{28}$ Cancer Epidemiology Unit, Nuffield Department of Population Health, University of Oxford, Oxford, UK; ${ }^{29}$ Yorkshire and Humber Research Network Consumer Research Panel, Sheffield, UK; ${ }^{30}$ Leeds General Infirmary, Leeds, West Yorkshire, UK and ${ }^{31}$ Liverpool and broadgreen Hospitals NHS Foundation Trust, Thomas Drive, Liverpool, Merseyside, UK

\section{ACKNOWLEDGEMENTS}

The views expressed are those of the authors and not necessarily those of the National Health Service, the NIHR or the Department of Health.

\section{AUTHOR CONTRIBUTIONS}

Substantial contributions to the conception of the study: L.W., K.A.C., M.W.R., A.B., M.B., C.M., J.L.M., S.J.W., A.R., N.M.L.B. Design of the work: L.W., K.A.C., M.W.R., M.B., J.L.M., S.J.W., A.R., N.M.L.B., C.M., M.B., T.C., K.J.P., D.R., T.G., J.G., T.R., R.A.A., G.R.H. Acquisition of data: L.W., C.M., J.L.M., M.B., M.W.R., K.A.C., T.C., K.J.P., K.L., A.E., A.T., 
K.L.C., C.H., M.C.W., M.A.G., M.Q.H., A.M.T., J.N., R.P., D.R., A.R., N.M.L.B., T.G., J.G., T.G.R., R.A.A., K.H. Analysis of data: L.W., S.E.W., M.B., E.H., N.M.L.B., S.J.W., C.M., J.L.M., M.B. Interpretation of data for the work: L.W., S.W., M.B., E.H., J.L.M., N.M.L.B., A.R. Data monitoring and ethics committee: A.M.T., M.A.G. and M.Q.H. Drafting the work or revising it critically for important intellectual content: A.R., N.M.L.B., M.W.R.R., E.H., J.L.M., M.B., S.J.W., K.A.C., S.E.W., G.R.H., M.B., K.L., A.E., T.G.R., C.M., T.C., K.J.P., A.B., K.L.C., A.T., R.A.A., J.W., R.S., T.G., D.R., J.G., K.H., C.H., M.C.W., J.N., R.P., M.A.G., M.Q.H., A.M.T., L.W. Final approval of the version to be published: A.R., N.M.L.B., M.W.R.R., E.H., J.L.M., M.B., S.J.W., K.A.C., S.E.W., G.R.H., M.B., K.L., A.E., T.G.R., C.M., T.C., K.J.P., A.B., K.L.C., A.T., R.A.A., J.W., R.S., T.G., D.R., J.G., K.H., C.H., M.C.W., J.N., R.P., M.A.G., M.Q.H., A.M.T., L.W. Agreement to be accountable for all aspects of the work in ensuring that questions related to the accuracy or integrity of any part of the work are appropriately investigated and resolved: A.R., N.M.L.B., M.W.R.R., E.H., J.L.M., M.B., S.J.W., K.A.C., S.E.W., G.R.H., M.B., K.L., A.E., T.G.R., C.M., T.C., K.J.P., A.B., K.L.C., A.T., R.A.A., J.W., R.S., T.G., D.R., J.G., K.H., C.H., M.C.W., J.N., R.P., M.A.G., M.Q.H., A.M.T., L.W.

\section{ADDITIONAL INFORMATION}

Ethics approval and consent to participate Ethics approval (NRES Committee London South East REC-IRAS: 12 LO 1808) and research governance approval were obtained. All patients (or their proxies, if cognitively impaired) gave written informed consent. The study was performed in accordance with the Declaration of Helsinki.

Consent to publish Not applicable.

Data availability The datasets generated during and/or analysed during the current study are available from the corresponding author on reasonable request.

Competing interests The authors declare no competing interests. Professors Stephen Walters and Thompson Robinson are National Institute for Health Research (NIHR) Senior Investigators, Jenna Morgan is a NIHR Clinical Lecturer and Kate Lifford is funded by the NIHR as part of this project. The views expressed in this article are those of the author(s) and not necessarily those of the NIHR, or the Department of Health and Social Care.

Funding information The study was sponsored by Doncaster and Bassetlaw Teaching Hospitals NHS Foundation Trust. Clinical Research Office, First Floor ' $\mathrm{C}$ ' Block, Doncaster Royal Infirmary, Armthorpe Road, Doncaster, DN2 5LT, UK. This paper presents independent research funded by the National Institute for Health Research (NIHR) under its Programme Grants for Applied Research Programme (Grant Reference Number RP-PG-1209-10071). The views expressed are those of the authors and not necessarily those of the NIHR or the Department of Health and Social Care.

The online version contains supplementary material available at https://doi.org/ 10.1038/s41416-021-01388-9.

Publisher's note Springer Nature remains neutral with regard to jurisdictional claims in published maps and institutional affiliations.

\section{REFERENCES}

1. Cancer Research UK. https://www.cancerresearchuk.org/health-professional/ cancer-statistics/statistics-by-cancer-type/breast-cancer/incidence-invasive Accessed January 2021.

2. Statistics OfN. Cancer survival in England: Patients diagnosed between 2010 and 2014 and followed up to 2015. (Newport, 2016).

3. Moller, H., Flatt, G. \& Moran, A. High cancer mortality rates in the elderly in the UK. Cancer Epidemiol. 35, 407-412 (2011).

4. Battisti, N. M. L., Wallington, M., Ring, A., Payne, S., Birch, R., Bomb, M. et al. Is age a barrier to chemotherapy? Rates of treatment in older patients treated with breast, colon and lung cancer in England in 2014: a national registry study. Ann. Oncol. 29, viii562-viii575 (2018).

5. Derks, M. G. M., Bastiaannet, E., Kiderlen, M., Hilling, D. E., Boelens, P. G., Walsh, $P$. M. et al. Variation in treatment and survival of older patients with nonmetastatic breast cancer in five European countries: a population-based cohort study from the EURECCA Breast Cancer Group. Br. J. Cancer 119, 121-129 (2018).

6. Khan, K., Seymour, M., Ring, A. \& Johnson, P. in Problem solving in older cancer patients (eds. Ring A., Mansi J., Harari D., Kalsi T., Selby P. J.) (Clinical Publishing, 2015)
7. Ring, A., Reed, M., Leonard, R., Kunkler, I., Muss, H., Wildiers, H. et al. The treatment of early breast cancer in women over the age of 70. Br. J. Cancer 105, 189-193 (2011).

8. Charlson, M. E., Pompei, P., Ales, K. L. \& MacKenzie, C. R. A new method of classifying prognostic comorbidity in longitudinal studies: development and validation. J. Chronic Dis. 40, 373-383 (1987).

9. Ottery, F. D. Definition of standardized nutritional assessment and interventional pathways in oncology. Nutrition 12, S15-S19 (1996).

10. Vigano, A. L., di Tomasso, J., Kilgour, R. D., Trutschnigg, B., Lucar, E., Morais, J. A. et al. The abridged patient-generated subjective global assessment is a useful tool for early detection and characterization of cancer cachexia. J. Acad. Nutr. Diet. 114, 1088-1098 (2014).

11. Mahoney, F. I. \& Barthel, D. W. Functional evaluation: The Barthel Index. Md State Med. J. 14, 61-65 (1965).

12. Lawton, M. P. \& Brody, E. M. Assessment of older people: self-maintaining and instrumental activities of daily living. Gerontologist 9, 179-186 (1969).

13. Folstein, M. F., Folstein, S. E. \& McHugh, P. R. "Mini-mental state". A practical method for grading the cognitive state of patients for the clinician. J. Psychiatr. Res. 12, 189-198 (1975).

14. Fayers, P. M., Aaronson, N. K., Bjordal, K., Groenvold, M., Curran, D. \& Bottomley, A. The EORTC QLQ-C30 Scoring Manual 3rd edn. (Cancer EOfRaTo, 2001).

15. Aaronson, N. K., Ahmedzai, S., Bergman, B., Bullinger, M., Cull, A., Duez, N. J. et al. The European Organization for Research and Treatment of Cancer QLQ-C30: a quality-of-life instrument for use in international clinical trials in oncology. J. Natl. Cancer Inst. 85, 365-376 (1993).

16. Sprangers, M. A., Groenvold, M., Arraras, J. I., Franklin, J., te Velde, A., Muller, M. et al. The European Organization for Research and Treatment of Cancer breast cancer-specific quality-of-life questionnaire module: first results from a threecountry field study. J. Clin. Oncol. 14, 2756-2768 (1996).

17. Johnson, C., Fitzsimmons, D., Gilbert, J., Arrarras, J. I., Hammerlid, E., Bredart, A. et al. Development of the European Organisation for Research and Treatment of Cancer quality of life questionnaire module for older people with cancer: The EORTC QLQ-ELD15. Eur. J. Cancer 46, 2242-2252 (2010).

18. Battisti, N. M. L., Herbert, E., Bradburn, M., Morgan, J., Wyld, L. \& Ring, A. Impact of chemotherapy for early stage breast cancer on quality of life in older adults: results from 2811 women enrolled in the Bridging the Age Gap study (\#2586) (European Society for Medical Oncology, 2020).

19. R Core Team. R: A language and environment for statistical computing. https:// www.R-project.org/. (2020).

20. Galea, M. H., Blamey, R. W., Elston, C. E. \& Ellis, I. O. The Nottingham Prognostic Index in primary breast cancer. Breast Cancer Res. Treat. 22, 207-219 (1992).

21. EuroQoL Research Foundation. EQ-5D-5L User Guide: Basic information on how to use the EQ-5D-5L instrument. (2019).

22. von Elm, E., Altman, D. G., Egger, M., Pocock, S. J., Gøtzsche, P. C. \& Vandenbroucke, J. P. The Strengthening the Reporting of Observational Studies in Epidemiology (STROBE) statement: guidelines for reporting observational studies. J. Clin. Epidemiol. 61, 344-349 (2008).

23. Ring, A., Harder, H., Langridge, C., Ballinger, R. S. \& Fallowfield, L. J. Adjuvant chemotherapy in elderly women with breast cancer (AChEW): an observational study identifying MDT perceptions and barriers to decision making. Ann. Oncol. 24, 1211-1219 (2013).

24. Bastiaannet, E., Liefers, G. J., de Craen, A. J., Kuppen, P. J., van de Water, W., Portielje, J. E. et al. Breast cancer in elderly compared to younger patients in the Netherlands: stage at diagnosis, treatment and survival in 127,805 unselected patients. Breast Cancer Res. Treat. 124, 801-807 (2010).

25. Buist, D. S., Chubak, J., Prout, M., Yood, M. U., Bosco, J. L., Thwin, S. S. et al. Referral, receipt, and completion of chemotherapy in patients with early-stage breast cancer older than 65 years and at high risk of breast cancer recurrence. J. Clin. Oncol. 27, 4508-4514 (2009).

26. Muss, H. B., Berry, D. A., Cirrincione, C. T., Theodoulou, M., Mauer, A. M., Kornblith, A. B. et al. Adjuvant chemotherapy in older women with early-stage breast cancer. N. Engl. J. Med. 360, 2055-2065 (2009).

27. Crivellari, D., Gray, K. P., Dellapasqua, S., Puglisi, F., Ribi, K., Price, K. N. et al. Adjuvant pegylated liposomal doxorubicin for older women with endocrine nonresponsive breast cancer who are NOT suitable for a "standard chemotherapy regimen": the CASA randomized trial. Breast 22, 130-137 (2013).

28. Leonard, R., Ballinger, R., Cameron, D., Ellis, P., Fallowfield, L., Gosney, M. et al. Adjuvant chemotherapy in older women (ACTION) study-what did we learn from the pilot phase? Br. J. Cancer 105, 1260-1266 (2011).

29. Im, S. A., Lu, Y. S., Bardia, A., Harbeck, N., Colleoni, M., Franke, F. et al. Overall survival with ribociclib plus endocrine therapy in breast cancer. N. Engl. J. Med. 381, 307-316 (2019).

30. Giordano, S. H., Duan, Z., Kuo, Y. F., Hortobagyi, G. N. \& Goodwin, J. S. Use and outcomes of adjuvant chemotherapy in older women with breast cancer. J. Clin. Oncol. 24, 2750-2756 (2006). 
Bridging The Age Gap: observational cohort study of effects of... A Ring et al.

31. Syed, B. M., Green, A. R., Ellis, I. O. \& Cheung, K. L. Human epidermal growth receptor- 2 overexpressing early operable primary breast cancers in older ( $\geq 70$ years) women: biology and clinical outcome in comparison with younger $(<70$ years) patients. Ann. Oncol. 25, 837-842 (2014).

32. Adjogatse, D., Thanopoulou, E., Okines, A., Thillai, K., Tasker, F., Johnston, S. R. et al. Febrile neutropaenia and chemotherapy discontinuation in women aged 70 years or older receiving adjuvant chemotherapy for early breast cancer. Clin. Oncol. (R. Coll. Radio.) 26, 692-696 (2014).

33. Ferreira, A. R., Di Meglio, A., Pistilli, B., Gbenou, A. S., El-Mouhebb, M., Dauchy, S. et al. Differential impact of endocrine therapy and chemotherapy on quality of life of breast cancer survivors: a prospective patient-reported outcomes analysis. Ann. Oncol. 30, 1784-1795 (2019).

34. Quinten, C., Kenis, C., Hamaker, M., Coolbrandt, A., Brouwers, B., Dal Lago, L. et al. The effect of adjuvant chemotherapy on symptom burden and quality of life over time; a preliminary prospective observational study using individual data of patients aged $\geq 70$ with early stage invasive breast cancer. J. Geriatr. Oncol 9, 152-162 (2018)

35. Todd, A., Martin, C., Morgan, J., Herbert, E., Bradburn, M., Burton, M. et al. Age specific recruitment and retention to a large multicentre observational breast cancer trial in older women: The Age Gap Trial. J. Geriatr. Oncol. S1879-4068(20) 30487-2. https://doi.org/10.1016/j.jgo.2020.10.015 (2020).

(i) Open Access This article is licensed under a Creative Commons Attribution 4.0 International License, which permits use, sharing adaptation, distribution and reproduction in any medium or format, as long as you give appropriate credit to the original author(s) and the source, provide a link to the Creative Commons license, and indicate if changes were made. The images or other third party material in this article are included in the article's Creative Commons license, unless indicated otherwise in a credit line to the material. If material is not included in the article's Creative Commons license and your intended use is not permitted by statutory regulation or exceeds the permitted use, you will need to obtain permission directly from the copyright holder. To view a copy of this license, visit http://creativecommons. org/licenses/by/4.0/.

(c) The Author(s) 2021 